\title{
Electrogravitic Propulsion System for a Satellite
}

\author{
Takaaki Musha
}

\author{
Advanced Science-Technology Research Organization, Yokohama, JAPAN \\ Foundation of Physics Research Center (FoPRC), Cosenza, ITALY. \\ E-mail: takaaki.mushya@gmail.com
}

corresponding author details: Takaaki Musha; takaaki.mushya@gmail.com

\begin{abstract}
Several methods for space propulsion, both pragmatic and hypothetical, have been developed each having its own drawbacks and advantages. In this paper, the possibility to propel a satellite by electrogravitic force is discussed. By theoretical calculation, this propulsion method can generate sufficient force to control the orbit of a satellite. It only uses electric energy generated by the use of solar panels and the satellite can travel perpetually around the Earth and any orbit near the Sun.
\end{abstract}

\section{Keywords: Space propulsion; Satellite; Electrogravity; Biefeld-Brown effect}

\section{INTRODUCTION}

All spacecraft require a method of propulsion. Several space propulsion methods, both pragmatic and hypothetical, have been developed each having its own drawbacks and advantages. The initial launch of a satellite to the desired orbit requires conventional liquid or solid propelled rocket engines with sufficient propulsive power to overcome Earth's atmosphere and achieve the high velocity necessary for a stable orbit. Interplanetary spacecraft may require such powerful conventional rocket engines but can also rely on less powerful but longer duration, higher ISP engines like ion thrusters or Halleffect thrusters. Satellite's, even once in a stable orbit, also require a reliable long duration method of propulsion to remain functional.

Even though a satellite is in orbit, it is subject to drag from the thin atmosphere and other forces which will degrade the orbit over time. Thus satellite's must be able to make small corrections to their orbit in order to maintain the orbit, called orbital station keeping [1]. Further, the satellite may require the ability to transfer from one orbit to another from time to time [2], be capable of maintaining a specific attitude with respect to the surface of the Earth, Sun or possibly some other astronomical object of interest [3], and due to component failure or other reasons may even need to be deorbited in a safe and controlled manner. In most cases, a satellite's ability to perform the task for which is was designed is over and its useful life has ended, when the propulsion system which allowed it to perform such adjustments to its orbit is exhausted or can no longer produce propulsion.

Currently satellites will often just use smaller versions of the chemical rocket engines or resistojet rockets for their propulsion. Some do use electric momentum wheels for attitude control, but these are subject to failure due to moving parts and limited in the extent of the corrections they can perform. Recently, satellites have begun to use electric propulsion, like ion thrusters for station keeping and adjusting their orbits, but such thrusters, although electrically powered, still have a limited supply of their required Xenon propellant. Ideally a propellantless form of electric propulsion would allow a satellite to perform its duties for a very long duration, greatly extending the useful lifetime of the satellite. Solar sails are one example of such a very low power, inexhaustible thrust [4], but this method is not well suited to an orbiting spacecraft and would be best applied to a very long duration interplanetary vehicle. There is a need for a new satellite propulsion system which has similar inexhaustible thrust, does not require propellant, has no moving parts and thus can provide propulsion for a very long duration.

In this paper, a new propulsion system which meets these requirements is proposed, which does not use propellant, has inexhaustible thrust and no moving part, giving similar advantages to a solar sail. This propulsion system utilizes the Biefeld-Brown effect, which can be called an electrogravity, and can provide propulsion for a long duration because it's only requirement is the electric power generated by the solar cell.

\section{ELECTROGRAVITIC PROPULSION SYSTEM}

This effect is known as the Biefeld-Brown effect; it encompasses electrokinetic effects, but is often claimed to have an electrogravitic component as well. The latter was reported by Thomas Townsend Brown $(1928,1929)$ to generate thrust without reliance on a surrounding medium (e.g., air) by applying high voltages to materials with high dielectric constants [5]. Recent electrogravitic tests in Canada and Japan have confirmed the electogravitic component of this effect [6]. Aviation photographer, Doyle R. Buehler, and Takaaki Musha, member of the Advanced Space Propulsion Investigation Committee under the Japan Society for Aeronautical and Space Sciences, also reported positive results. This is a primitive field propulsion system which can be used to propel only small space probes as pointed out by N. Iwanaga in his paper [7].

The author obtained the following formula for the electrogravitic effects induced on a dielectric material from a weak field approximation of Einstein's general relativity theory [8]. 


$$
E_{g} \approx-Z \sqrt{4 \pi \varepsilon_{r} \varepsilon_{0} G} \cdot E=-8.62 \times 10^{-11} Z \sqrt{\varepsilon_{r}} \cdot E
$$

where $Z$ is an atomic number of the dielectric material, $\mathcal{E}_{r}$ is the specific inductive capacity, $\mathcal{E}_{o}$ is a dielectric constant of the vacuum, $G$ is the gravitational constant and $E$ is the applied electric field to the capacitor.

B. Ivanov of the Institute for nuclear Research and Nuclear Energy in Bulgaria also found the similar equation deduced from Weyl Majumdar-Papapetrou solutions of the general relativity theory [9-11], which is equivalent (modulo Z) to the author's equation, as shown in the following figure.

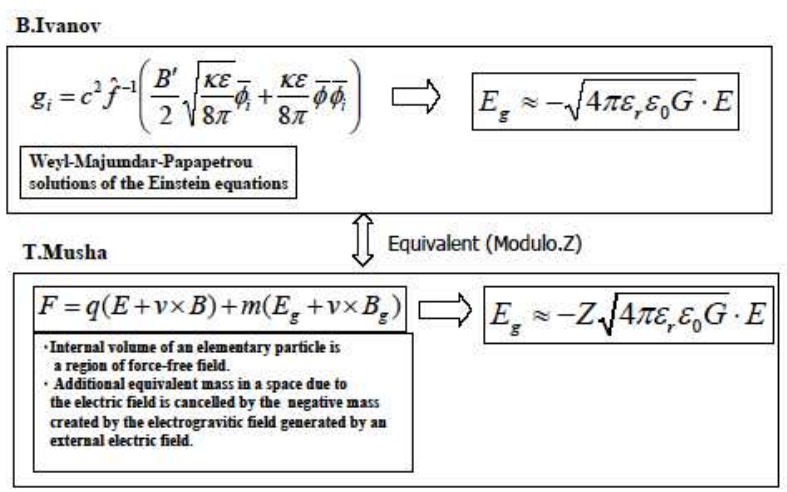

FIGURE 1: Electogravitic equation derived by Ivanov and Musha

The force generated by high voltage can be written as follows from Eq.(1).

$$
F=m E_{g}=8.62 \times 10^{-11} \mathrm{Zm} \sqrt{\varepsilon_{r}} \frac{V}{d},
$$

where $m$ is the weight of the dielectric material, $V$ is the applied voltage and $d$ is the thickness of the dielectric material. We consider a simple electric circuit consisted of a resistance and a capacitance as follows;

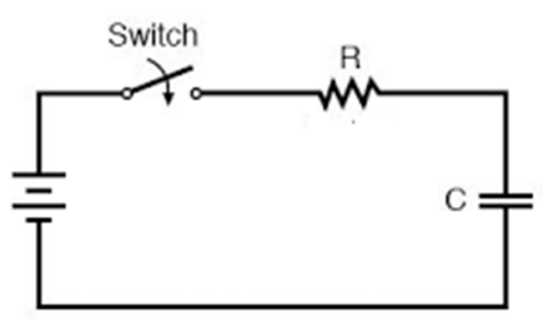

FIGURE 2: Switching circuit with a resistance and a capacitance

When the switch is on, the electric current is produced as shown

$$
i=\frac{V}{R} \exp \left(-\frac{t}{C R}\right)
$$

where $V$ is a voltage, $R$ is the resistance of the circuit, $C$ is the capacitance of a capacitor and $t$ is a time.

Then the instantaneous electric power generated on the capacitor becomes

$$
p_{C}=\frac{V^{2}}{R}\left(1-\exp \left(-\frac{t}{C R}\right)\right) \cdot \exp \left(-\frac{t}{C R}\right)
$$

Hence the total electrostatic energy stored in the capacitor received from the power source becomes

$$
W_{C}=\int_{0}^{\infty} p_{c} d t=\frac{1}{2} C V^{2},
$$

As the capacitance can be shown as $C=\frac{\mathcal{E}_{r} \mathcal{E}_{0} S}{d}$, where

$S$ is an area of the capacitor, we have

$$
\alpha=F / M=8.62 \times 10^{-11} Z \frac{m}{M} \sqrt{\frac{2 W_{C}}{\varepsilon_{0} d S}}=2.9 \times 10^{-5} Z \frac{m}{M} \sqrt{\frac{2 W_{C}}{d S}}
$$

from Eq. (2), where $M$ is the total weight of the satellite.

For a series capacitor as shown in Fig. 3, we have $C=n C_{0}$, where $n$ is a number of the series capacitor and $C_{0}$ is the capacitance of a single capacitor.

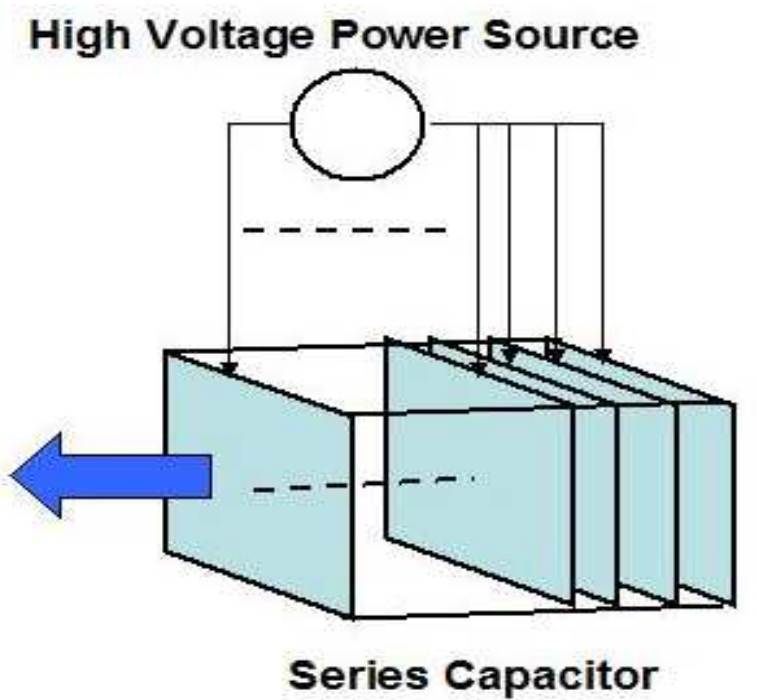

FIGURE 3: Series capacitor connected with a high voltage source

Then we have an acceleration given by

$\alpha=2.9 \times 10^{-5} Z \frac{m n}{M} \sqrt{\frac{2 W_{C}}{n d S}}=2.9 \times 10^{-5} Z \frac{m}{M} \sqrt{\frac{2 n W_{C}}{d S}}$

for a capacitor, which is consisted of a $n$ pieces series capacitor, which total mass is $m$. By using the equation which the author derived, Iwanaga analyzed the effectiveness of electrogravity as a propulsion system in his paper [7] and he estimated that the thrust obtained by electrogravitic propulsion is much better than that of arcjet propulsion system as shown in TABLE 1.

TABLE 1: Thrust of some propulsion systems and Electrogravity

\begin{tabular}{|l|l|}
\hline Propulsion System & Thrust (N) \\
\hline Jet engine & $2 \times 10^{5}$ \\
\hline Chemical fuel rocket & $2.45 \times 10^{5}$ \\
\hline Arc-jet & $1.50 \times 10^{-1}$ \\
\hline Nuclear power & $8.82 \times 10^{5}$ \\
\hline Photon rocket & 3.3 \\
\hline Electrograviy* & 100 \\
\hline
\end{tabular}

${ }^{*}$ Calculated for mass $=100 \mathrm{Kg}$, diameter $/$ height ratio $=100$, $\varepsilon_{r}=5$ and $E=7 \times 10^{8} \mathrm{~V} / \mathrm{m}[7]$ 
He considered it would be profitable to apply this propulsion method to small space vehicles. From the comparison made in Table. 1 , it is clear that the electrogravitic propulsion system is more advantageous than those nowadays in use.

\section{DESIGN CONCEPT OF THE ELECTROGRAVITIC PROPULSION SATELLITE}

From the theory delineated in the former section, we can propose the following structure of the satellite as shown in FIGURE 4.

The satellite consists of a solar panel, a high-voltage pulse generator, a series capacitor composed of a dielectric material to generate a thrust and the control device.

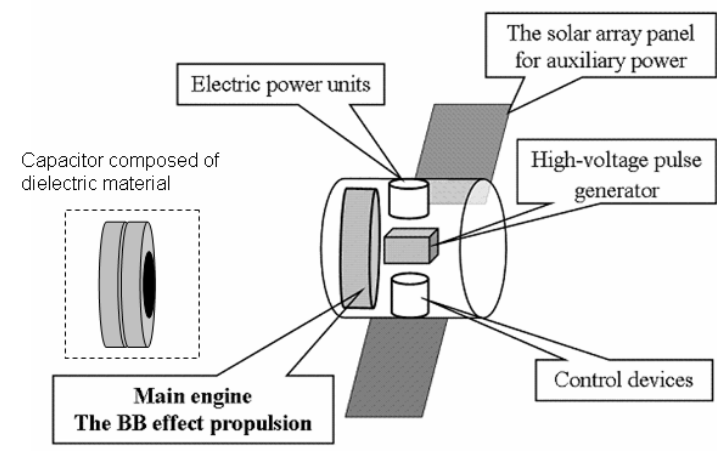

FIGURE 4: Concept of the satellite propelled by the electrogravitic force

The components to produce the high voltage which is applied to the capacitor (or condenser) are shown in Fig. 5. This electric circuit can be used to produce a high voltage electric pulse.

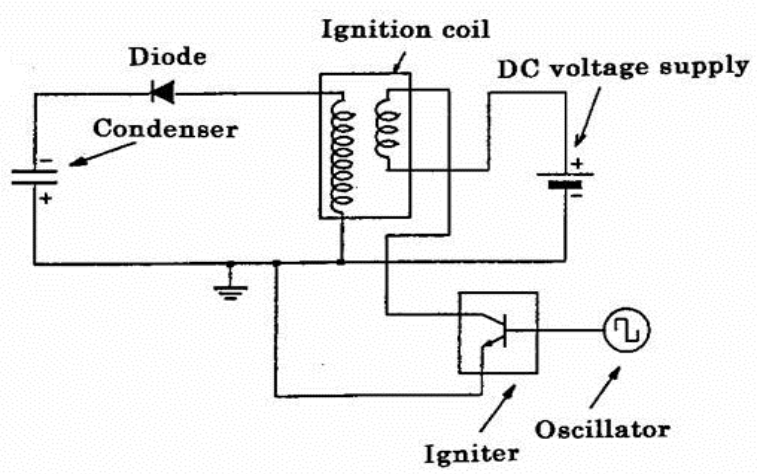

FIGURE 5: Electric circuit to produce a high voltage suppled to the capacitor

For example, if we use four solar panels, the electric power of each solar panel is $250(\mathrm{~W})$, the total electric energy becomes $W_{C}=250(W) \times 1(\mathrm{sec}) \times 4=1000(\mathrm{~J})$

If we let $n=100, d=2 \times 10^{-3}(\mathrm{~m}), S=10^{-2}(\mathrm{~m})$, $m / M=0.1$, and $Z=10$, the acceleration generate by electrogravitic effect is $\alpha=2.9\left(\mathrm{~m} / \mathrm{sec}^{2}\right)$ from Eq. (7).

This value is sufficient for orbital station-keeping and some degree of attitude control.

This satellite controlled from the Earth could change its orbit by using this electrogravitic thrust. Such a propulsion system could also make an interplanetary voyage within the solar system with inexhaustible thrust similar to solar sails, but provides stronger thrust than a solar sail.

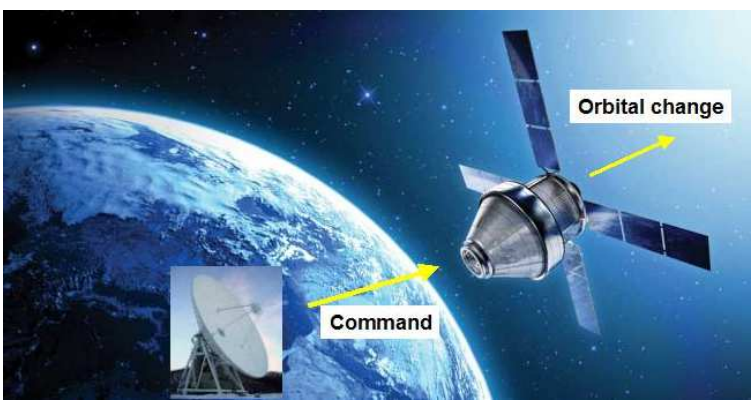

FIGURE 6: Concept of the satellite using Electrogravitic propulsion

\section{CONCLUSION}

Space propulsion or in-space propulsion exclusively deals with propulsion systems used in the vacuum of space. Several methods, both pragmatic and hypothetical, have been developed each having its own drawbacks and advantages. In this paper, the possibility to propel a satellite by electrogravitic force is discussed. Utilizing theoretical calculation, this propulsion method can generate sufficient force to control the orbit of a satellite. This space propulsion method only uses the electric energy generated by solar panels and the satellite can travel perpetually around the Earth and any orbit near the Sun.

\section{ACKNOWLEDGEMENT}

The author appreciates Mr. Grant Hayman of OVAL Space Technologies in Canada for his help and suggestions on satellite and rocket technology, which greatly improved this paper.

\section{REFERENCES}

[1] Phllips, T., Solar S'Mores, NASA,2007-07-30 (May 30,2000)

[2] Olsen, C., Hohman Transfer \& Plane Changes, NASA, 2007-07-15 (Sept.21, 1995)

[3] Hess, M, Martin, K. K., Rachul, L. J., Thrusters Precisely Guide EO-1 Satellite in Space First, NASA, 2007-12-06 (Feb.7,2002)

[4] Anonymous, Basic Facts on Cosmos 1 and Solar Sailing, The Planetary Society (July 3,2007)

[5] INTEL, Toward Flight without Stress or Strain or Weight, Interavia, XI,(1956) pp.373-374.

[6] Musha, T., Explanation of Dynamical Biefeld-Brown Effect from the Standpoint of ZPF Field, JBIS, Vol.61, (2008) pp.379-384.

[7] Iwanaga, N., Review of Some Field Propulsion Methods from General Relativistic Standpoint, Space Technology and Applications International Forum, The American Institute of Physics, Issue 104, 2012, pp.27-30.

[8] Musha, T, The possibility of strong coupling between electricity and gravitation, Infinite Energy, Isuue.53 (2004) pp.61-64.

[9] Ivanov, B.V., Weyl Electrovacuum Solutions and Gauge Invariance: Preliminary research for gravity control, Journal of Space Exploration, Vol.2(1), (2013) pp.19-51.

[10] Ivanov, B.V., Strong gravitational force induced by static electromagnetic fields, arXiv: gr-qc/0407048vl, July 13, 2004.

[11] Ivanov, B.V., On the gravitational field induced by static electromagnetic sources, arXiv: gr-qc/0502047 vl, February 10, 2005. 